\title{
Smartphone Applications for the Radiologist
}

\author{
Hasanein Al-Hasani ${ }^{1 *}$, Hamid Abboudi ${ }^{2}$, Tishi Ninan ${ }^{1}$, Behnam Shaygi ${ }^{1}$, Carl Roobottom ${ }^{1}$ \\ ${ }^{1}$ Department of Radiology, Plymouth Hospitals NHS Trust, Derriford Hospital, Plymouth, UK \\ ${ }^{2}$ Department of Radiology, Royal Sussex County Hospital, Brighton, UK \\ Email: "hasanein@doctors.org.uk
}

Received October 21, 2013; revised November 21, 2013; accepted November 28, 2013

Copyright (C) 2013 Hasanein Al-Hasani et al. This is an open access article distributed under the Creative Commons Attribution License, which permits unrestricted use, distribution, and reproduction in any medium, provided the original work is properly cited. In accordance of the Creative Commons Attribution License all Copyrights (C) 2013 are reserved for SCIRP and the owner of the intellectual property Hasanein Al-Hasani et al. All Copyright (C) 2013 are guarded by law and by SCIRP as a guardian.

\begin{abstract}
Smart phone applications (apps) for radiologists are on the rise. Not only do they assist the radiologist in reference to information but they can also aid in their day-to-day functioning too most notably through image viewing apps known as Digital Imaging and Communications in Medicine (DICOM) viewers. These kinds of apps signal a transition towards an increasingly mobile medical environment in what could be an exciting but cautious time for radiologists as these apps are not without their own concerns. In this review we provide basic information on how to create an app, highlight the current state of play of pivotal radiology apps and discuss the legal issues surrounding them.
\end{abstract}

Keywords: Smartphone; Radiology; Applications

\section{The Rise of Smartphones and Radiology Apps}

The ability to access information at will has long been the dream of human beings and several major developments in the history of mankind have been driven by this aspiration. Invention of paper, printing, computers and the internet has brought this dream closer but none more so than the newest gifts of technology, the "smart" devices. Smartphones, together with tablets are generally regarded as hand held computers rather than simply mobile telephones and the development of downloadable applications (apps) has created a vibrant new industry. They have put repositories of books, online knowledge, images and communication literally at our fingertips. While the internet transformed from "information superhighway" to the "world wide jungle" in about a decade, smartphone apps have managed to proliferate extensively in less than half of the time since the introduction of Apple's iTunes App Store in July 2008.

The visual nature of radiology makes the specialty a promising area for smartphone app development. A recent study identified 321 radiology apps, the majority of which (88\%) were available from either the Apple (54\%) or Android platforms $(34 \%$, ) which is an open source operating system that is run by a growing number of mo-

\footnotetext{
*Corresponding author.
}

bile phones including the popular Samsung, Sony and HTC devices. Teaching (e.g. flashcards, questions, revision guides/notes, textbooks, cases, tutorials/talks, etc.) and reference (e.g. guidelines, cancer staging, radiology positioning guides, scanning protocols, glossaries, calculators, converters, etc.) apps count for $79 \%$ of radiology apps [1]. A survey of radiology trainees across the US in 2011 showed that $74 \%$ owned a smartphone [2]. However, there are no equivalent data for radiologists in the UK currently. There are also no figures of projection of growth of radiology apps but if they are expected to grow at the same rate as medical apps as a whole they will grow by $25 \%$ annually for the next five years [3].

\section{Creating an App Yourself}

Radiologists can create a web-based app (web app) using the iWebKit app for the iPhone which enables you to create a web app emulating the look and feel of native iPhone apps (Figure 1). It is a free app using basic Hyper Text Markup Language (HTML, the authoring language used to create web pages), Cascading Style Sheets (CSS, for defining the visual presentation of HTML) and JavaScript (scripting language which interacts with HTML codes to design interactive sites.) Even for those without basic web skills it is still possible to create a fairly complex web app with the help of a user guide that 


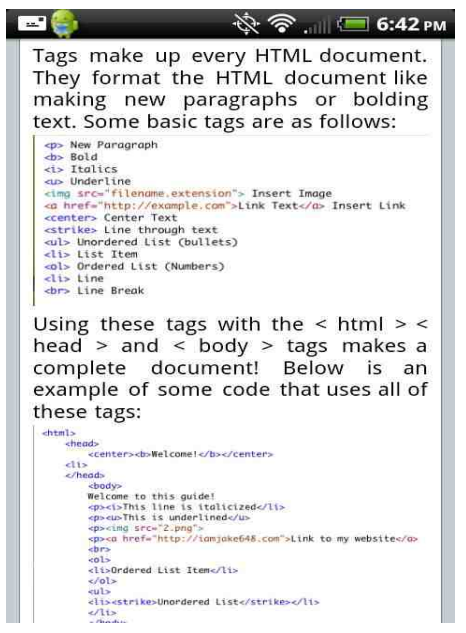

Figure 1. iWebkit user guide.

comes with iWebkit. Preloaded HTML codes are provided that can be customised for example including a link to a radiology image with associated text.

You can create several user interface elements such as forms, buttons, text fields, menus and lists and these are constantly being updated. If designing a web app for a department, their own icons for thumb-nails can be added for further personalisation. Popular analytics sites such as Google Analytics can be utilised by incorporating codes, this will generate detailed statistics about how visitors actually interact with your web app so you can make informed design improvements [4]. There is an option to create $\mathrm{QR}$ codes, a two-dimensional barcode for which the iPhone user can scan using their iPhone camera to provide a link to supplemental material whilst creating a tech savvy image. This QR code can even be displayed in advertisements on the wall in the patient waiting room for patients to gain electronic access to your web app.

\section{Categorisation of Radiology Apps}

Apps can broadly be divided into web apps and native apps. Web apps act as a portal to retrieve and display information that is stored on a server in the worldwide web. Native applications are stored on the device. The drawback of web applications is that you need Wi-Fi or third generation (3G) connection to access the data. Native applications are limited by the available storage on the device but you do not have to be connected to the internet to be able to view the information. Some applications are a combination of both. For the purpose of this article however we have classified radiology apps into three different categories as follows.

\subsection{Viewing Software}

There is an obvious "cool" factor having apps whereby you are able to use intuitive tools on your phone to view radiology images, but outweighing this is the demand from users who want to access Digital Imaging and Communications in Medicine (DICOM) images on the move or from remote locations to use for collaboration, consultation and teaching. Teleradiology can be used to gain rapid opinions from senior colleagues when they are off site, allowing early intervention and potentially better patient outcomes. A universally utilised DICOM viewer paves the way for its corresponding cloud system to be a method of storage and enabling communication outside of the local network.

Smartphones cannot currently meet the minimum specifications for medical display. According to the Royal College of Radiology (RCR) a screen resolution of $\geq 1280 \times 1024$ pixels, screen size of $\geq 42 \mathrm{~cm}$ and the option of comparing serial examinations side by side are the minimum standards for primary diagnostic displays [5]. In comparison, the iPhone 5's display has $1136 \times 640$ pixels and a $10.2 \mathrm{~cm}$ screen and cannot compare images side by side [6]. In contrast to smartphones, tablet devices have larger displays with better resolution and therefore may be better suited for mobile review of DICOM images.

PACS workstations display systems are carefully selected and strictly monitored, maintained, and calibrated to ensure adequate image quality [7]. Smartphones looking to replace workstations being used for primary diagnosis would have to be part of similar quality assurance programmes and currently there is no scope for this. In addition there is difficulty in performing measurements on a small screen using fingers, increased strain on the eyes and a negative impact of ambient light on image quality. Users cannot dictate reports which would increase the viability of this product. There is also the issue of speed, there is limited access in most UK hospitals to Wi-Fi network and this renders the time taken to transfer the $1 \mathrm{~mm}$ slices to permit multiplanar reformatting considerably longer limiting the usefulness of these apps $[8,9]$.

The US Food and Drug Administration (FDA) approved 3 out of 29 currently available DICOM viewers for interpretation of medical images on mobile devices after undergoing several performance tests including measured luminance, image quality (resolution) and noise according to international standards and guidelines. They are however clear that they can only be used for this purpose where no access to a workstation is available or when doctors want to analyse images on-the-go $[1,10]$. They are not intended to replace workstations. With this in mind and accompanied by the hardware differences mentioned above, these DICOM viewers on smartphones are currently more suited for reviewing images (secondary interpretation) usually in conjunction with a report as opposed to primary interpretation [5]. 
An example of a DICOM viewer app is Mobile MIM (Figure 2). As well as being the first of its kind to undergo FDA approval in 2011 for viewing DICOM images in different modalities (including CT, MRI, nuclear medicine scans, diagnostic X-ray and ultrasound) it also has a European seal of approval as a medical device. Before the FDA labelled it a class III medical device (a highly regulated 'high risk' medical device) it won the Apple Design Award for "Best iPhone Healthcare \& Fitness Application" in 2008 [11]. Mobile MIM has introduced image intensity values, distance measurement display measurement lines, annotations and regions of interest. It also performs multiplanar reconstructions out of the box. Provided you configure a MIMpacs server in the imaging department to cache a subset of PACS data, images from the hospital can be sent over a secure network to the iPhone for viewing on the Mobile MIM app [12]. Neuroradiologists looked at 120 consecutive non-contrast CT brain scans and $70 \mathrm{CT}$ angiograms on the iPhone and iPod version of this app and results showed that there was a $94 \%$ to $100 \%$ rate of accuracy for diagnosing acute stroke compared with a PACS workstation [13]. Mobile MIM is paired with the MIM Cloud to enable a viewing and sharing web application respectively.

MIM Cloud as the name suggests is a cloud-based software that enables sharing of DICOM studies so users can maintain access whilst off site and do not require access to a PACS server. It enables easy sharing of information between professionals [14]. This reduces the chances of images getting lost through $\mathrm{CD}$ exchanges and scans being repeated unnecessarily especially in cases of patients who change doctors and hospitals for personal or medical reasons.

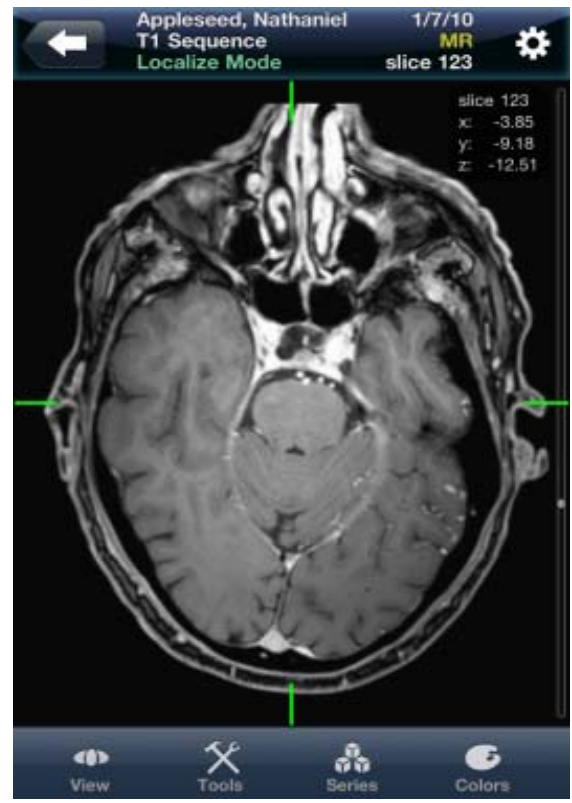

Figure 2. Mobile MIM app.
Osirix is mainly for use on Macintosh, this version (Osirix MD) is FDA approved for diagnostic use but the iPhone versions of the software by the same developers are not approved for diagnostic use. They can however be used to maintain personal libraries of interesting images and for teaching [15]. A study performed at the University of Virginia using Osirix on an iPhone suggests this app can be used for a quick diagnosis of appendicitis on CT as 99 percent of the time they correctly diagnosed acute appendicitis with one false negative and no false positives compared with reporting on a PACS workstation [16]. In another study, radiologists utilised Osirix display software on the iPhone for reviewing CT and MR images. They were as accurate as those reading images from PACS workstations with overall matching interpretations of 90 out of the $93 \mathrm{CT}$ and MRI exams with partial matching in the remaining 3 cases. Radiologists actually found more pathology was detected on the smartphones than on workstations as they searched more intensively at the expense of eye fatigue according to the participants [17].

Resolution MD in April 2013 is the first DICOM app that has received FDA clearance for diagnostic medical image viewing on Android mobile devices. In 2011 it was cleared for use on iPhone. ResolutionMD like Mobile MIM is also CE (Conformité Européenne) marked for distribution in Europe and licensed by Health Canada [18].

\subsection{Reference, Teaching and Logbook Apps}

The Radiology Assistant is an app for iPhone and more recently Android phones that provides users with peerreviewed educational articles on a wide variety of radiological topics [19]. Topics focus mainly on common pathology, in most cases there will be an image, graph or table followed by explanatory text. The images are very clear with arrows pointing at important features and diagrams where necessary. All content is available locally without internet connection. This can be a great advantage in the hospitals where internet connections can be sporadic and information is needed instantly [9]. Interactive encyclopaedias like these can make studying more applicable as you have to scan through the images making it more relevant as opposed to passively reading through text. An auto-notification feature was recently added to download new content. Other examples of similar interactive encyclopaedias include Radiology 2.0: one night in the ED and iRadiology.

Radiation Passport is an iPhone app aimed to educate the healthcare professional about the radiation exposure one can receive and the potential risk factors associated with different imaging modalities. It is based on international published scientific journal papers and data [20]. It also provides an estimated risk of developing malignancy 
as a result of the amount of x-ray exposure and accumulated dose. The app includes average radiation dose values for over 140 examinations and procedures and allows individuals to enter custom values to calculate total radiation exposure. The app also takes into account other factors such as the patient's age and gender, and provides extensive background information section with links to relevant studies and reports.

SonoAccess is an iPhone app consisting of a comprehensive library of clinical and instructional ultrasound videos and documents available on-demand. Within the SonoAccess application, you can customise your user profile to generate a recommended list of videos, and share any document or video with a colleague or friend [21].

Rad-Rx is an Android app made for radiologists, radiographers and emergency personnel. The Rad-Rx app provides quick and convenient access to algorithms for the treatment of allergic responses to intravenous contrast. It also includes advice on how to dose and administer iodinated and gadolinium-based contrast for patients with renal insufficiency [22].

While most people do not think of it as such, some websites such as Google Maps are actually web apps that reside on a remote server and interact with the user within the confines of a web browser. Similarly myRSNA subsection of RSNA.org, for which membership to the Radiological Society of North America (RSNA) is required, is a personalised online portal which enables users to receive full access and regularly updated information from the Radiology and RadioGraphics journals where users can access the latest and previous editions of the journals [23]. They also receive updated information about RSNA news articles, job opportunities, annual meeting content and more. Users can upload files of any type to a personal online directory of up to $1 \mathrm{~GB}$ of storage and access those files from any computer in the world via myRSNA. European Radiology, an official publication of the European Society of Radiology is available as a free app for the iPhone and Android with the latest developments in radiology and extensive research [24].

iRefer from The Royal College of Radiologists is a multi-platform reference app that provides guidelines for physicians in the selection of the most appropriate imaging investigation or intervention for a given diagnostic or imaging problem based on the best available evidence, together with expert medical and radiological opinion (Figure 3). It is fully functional when offline so no active data connection required [25].

Radiology Logbook Plugin of the Universal Logbook App is a computerised version of the Royal College of Radiologists' trainee log book for the iPhone designed by Consultant Radiologist Dr Raj Burgul [26]. It

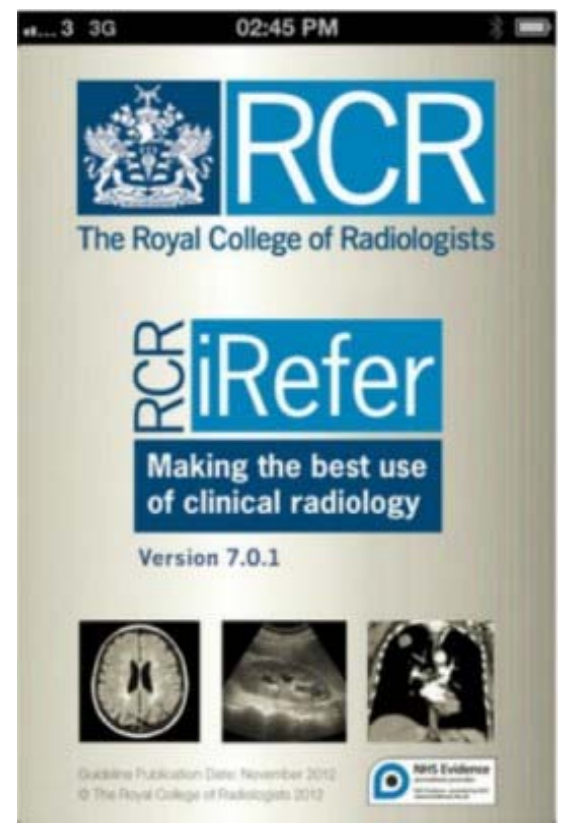

Figure 3. iRefer app.

is an on-the-go portfolio useful for trainees and is also available for other specialities. It allows for bidirectional iPhone import and export which automatically corrects the available data. Fields include indirect and direct supervision for each responsibility and summary tables, which display supervision levels. Trainees can therefore keep a portable record of cases and procedures protecting them against losing bits of paper. It also encourages continuous updating so the trainee can be more organised.

\subsection{Smartphone Based Ultrasound Device}

MobiUS by Monisante is an FDA approved hand-held mobile ultrasound probe attached to a Toshiba smartphone with the MobiUS app installed (Figure 4). This smartphone is not for personal use and is strictly for operating the ultrasound only and currently you cannot install the app on your iPhone or Android phone [27,28]. The hardware won the "Global Mobile Award for Best M-Health Innovation" at Mobile World Congress in Feb 2011. It gained a huge array of interest in its device among physicians in rural parts of the US and around the world where the technology is not widely available and as it is battery powered it can work in areas without reliable power [29]. Ultrasound images taken can be transferred in precisely the same way as photos taken using the smartphone's camera utilising the cellular network and Wi-Fi capabilities of the smartphone to send images remotely for diagnosis, second opinion or to PACS. They have similar useful tools like labelling lesions with the diagnosis in question with the added benefit of being extremely portable and very affordable compared to traditional ultrasound systems [28]. 


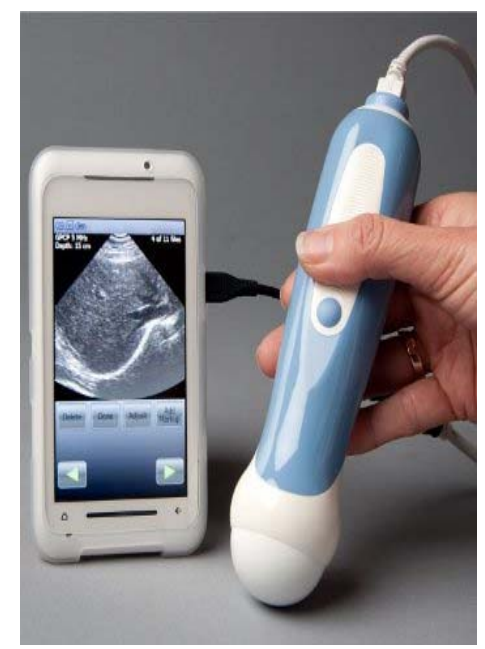

Figure 4. Mobisante ultrasound device.

\section{Legal Matters Surrounding Radiology Apps}

Apple has an approval process before an app becomes available on their store, the purpose of which is to ensure security, safeguard against inappropriate content and to search for software bugs. The review process does not check the qualifications of the developer, calculations or recommendations [30]. In theory it is up to governing bodies and accreditation groups to regulate apps classified as medical devices based on their intended use. In the US, congress passed a FDA Safety and Innovation bill in July 2012 and draft guidelines were issued. It was not until September 2013 when final guidance on mobile medical applications was released. It states that the FDA will apply its regulatory authorities to select "mobile medical applications" for regulation. These are defined as software applications run on a mobile platform that are either used as an accessory to a medical device or transforms a mobile platform into a regulated medical device. Not all apps are deemed necessary for regulation, for example those which act solely as reference or teaching guides which form the majority of medical apps. Before the final guidelines were released around 100 medical apps have received FDA approval but more approved apps are emerging [31]. The process of undergoing official regulation can be both time-consuming and expensive. According to the Government Accountability Office, the FDA takes about 6 months to approve a medical device that is similar to an existing product and 20 months to approve a brand new device [32].

The governing body responsible for the regulation of medical devices in the UK is the Medicines and Healthcare products Regulatory Agency (MHRA). To date only one app that is publically available for download has been registered as a medical device with the MHRA in the UK and that is Mersey Burns, a clinical tool for estimating burn area percentages and determining fluids to be prescribed accordingly. In the EU every registered medical device must carry the CE mark but there is currently no consensus between member states of what a medical device entails as each has its own regulatory framework of the Medical Devices Directive (MDD) which ensures safety and performance of medical devices throughout the EU [33].

Regulations are an important consumer safeguard and without question send a strong market message of user confidence. They are however in their infancy and regulators face an uphill task to approve existing apps along with those apps that are in development. Therefore in this current situation the ultimate responsibility lies with the individual professional user. They should make sure that the applications are used in a professional capacity and have ideally undergone the appropriate peer reviews but this can be non-existent because in a recent study only $44 \%$ of radiology apps acknowledged medical professional or society input. In addition relying on customer feedback based on ratings can also be minimal or lacking [1].

It is not only the approval process where the legal system lags behind but also in the advent of confidentiality and privacy of patient data from visualisation of radiological images on smartphones, so-called teleradiology through DICOM apps and their associated cloud technology. Concerns are perhaps more important with smartphones because their portability adds vulnerability to losing patient data as real-time imaging has the potential to be stored on an iPhone. Many doctors do not have passwords on their phones, access unsecure Wi-Fi networks and most do not have a formal safe-keeping strategy at their work, for example the inability to wipe out the data from an employee's personal device should it be lost or stolen [34]. This can be troublesome if not regulated, especially if targeted toward medical students still undergoing training on confidentiality at medical school. One study found that one in 10 UK medical students exhibited frank violations of patient confidentiality on social networking applications and mobile applications. In 2004, the General Medical Council described the importance of appropriate arrangements for the security of personal information when stored electronically. The General Medical Council states that patients have a right to confidentiality. Furthermore, trust between doctor and patient is central and is something that has been a privilege for centuries to those practicing medicine.

All personal identifiable data are regarded as protected by the holding organization, which has a duty to enforce confidentiality. In the US, the privacy rule of the Health Insurance Portability and Accountability Act of 1996 was enforced in 2003 to protect health information to maintain confidentiality. In the UK, there are no specific laws 
governing mobile handset use and patient confidentiality, but we currently have a legal requirement determined by common law, the Data Protection Act 1998 and the Freedom of Information Act 2000. Data that are regarded as confidential in nature are protected under the notion that the confidant (doctor) can disclose information only with consent from the confider (patient). The National Health Service is required to also abide by the National Health Service code of practice for confidentiality. There are six Caldicott principles related to transferring and collecting identifiable information. These principles govern the use of patient information, and every health professional using it needs to be aware of it. We must also consider the legal risk to health care trusts that do not make adequate attempts to prevent medical identity theft. Medical identity theft includes identifiable information, which can include birth date and patient addresses. Information can be sold to other parties and used for fraudulent purposes [35].

We embrace the emergence of mobile technology for radiologists, provided that there are more concise laws governing their use in the workplace. With the surge in app developers, the future impact remains to be seen.

\section{The Future of Apps in Radiology}

Rapid advancement in web browsers and software technologies dubbed web 2.0, or informally defined as the "participatory web" has occurred that could conceivably displace the central role of apps in the future [36]. In effect, the rise of a social, collaborative web through wikis, blogs and newsfeeds means that research, expertise and even software development are continuing to be group efforts. Radiologists will continue branching out to other radiologists who are active in the online community through social networks such as Radiolopolis, RadRounds, LinkedIn, Plaxo and it would be wrong for us at this point not to mention Facebook. This will enhance the quality of professional networks by simplifying the process of discovering contacts, posing questions, and sharing expertise to offer a whole new medium for peer-reviewed publication.

Radiology is becoming a notoriously competitive specialty, an app to aid prospective applicants on how best to approach upcoming interviews and information for current trainees on career progression in radiology would be useful. Other useful apps would be an interventional radiology specific manual app that would help trainees become more familiar with the procedures and reinforce the skills learned in the interventional suite.

A survey of radiology trainees across the US published in the American Journal of Radiology in 2011 showed that time dedicated to radiology education using electronic devices and online resources for their education is roughly equal to that from printed textbooks [2]. Not least because their favourite textbooks are available in ebooks which will make carrying heavy backpacks laden with radiology textbooks a thing of the past, this is beneficial not least for your back but also studying during travel or at the workstation. In the future, when or if this current economic climate ends, we may expect to see deaneries incorporate a system whereby they pay trainees for iPhone ownership and compulsory radiology apps to further officialise the use of iPhones for education in keeping with the times.

\section{Conclusion}

In conclusion, radiology has a good selection of useful apps which can be utilised by senior radiologists and those in training. With the increasing number of app developers, there is still scope for future developments and there is no reason why radiologists themselves cannot be at the forefront of app development. Limitations of image interpretation using smartphones are a drawback of DICOM viewing apps. The ethical and legal issues pertaining to the expansion of smartphone apps require further investigation.

\section{REFERENCES}

[1] M. A. Rodrigues, A. Visvanathan, J. T. Murchison and R. R. Brady, "Radiology Smartphone Applications; Current Provision and Cautions," Insights Imaging, Vol. 4, No. 5, 2013, pp. 555-562. http://dx.doi.org/10.1007/s13244-013-0274-4

[2] Health Imaging, "JACR: Survey Shows Radiology Education Going Mobile," 2012.

http://www.healthimaging.com/topics/health-it/jacr-surve $\mathrm{y}$-shows-radiology-education-going-mobile?page $=0 \% 2 \mathrm{C}$

[3] Health Data Management, "Kalorama Tracks Mobile Medical App Market," 2012.

http://www.healthdatamanagement.com/news/mobile-me dical-apps-applications-market-kalorama-44597-1.html

[4] HTML Goodies, "iWebkit iPhone Development Framework to Create Web Apps," 2013.

http://www.htmlgoodies.com/beyond/webmaster/toolbox/arti cle.php/3896706/Using-the-iWebkit-iPhone-Development-Fr amework-for-Creating-Web-Apps.html

[5] The Royal College of Radiologists, "Picture Archiving and Communication Systems (PACS) and Guidelines on Diagnostic Display Devices," 2012.

[6] Apple Inc., "iPhone," 2012.

http://www.apple.com/iphone/specs.html

[7] E. Seto, et al., "Image Quality Assurance of Soft Copy Display Systems," Journal of Digital Imaging, Vol. 18, No. 4, 2005, pp. 280-286. http://dx.doi.org/10.1007/s10278-005-6705-0

[8] J. E. Wilting, "Technical Aspects of Spiral CT," MedicaMundi, 1999.

http://www.healthcare.philips.com/pwc_hc/main/about/as sets/Docs/medicamundi/mm_vol43_no $1 / \mathrm{mm}_{-}$vol43_no1_ 
article_technical_aspects_of_spiral_ct.pdf

[9] eHealth Insider, "NHS Hack Day Presses for Free Wi-Fi," 2013.

http:/www.ehi.co.uk/news/ehi/8551/nhs-hack-day-presse s-for-free-wi-fi

[10] E. Jefferson, "FDA U.S. Food and Drug Administration, News \& Events," 2011.

http:/www.fda.gov/NewsEvents/Newsroom/PressAnnou ncements/ucm 242295.htm

[11] Apple Insider, "FDA approves iPad, iPhone radiology app for mobile diagnoses," 2011. http://appleinsider.com/articles/11/02/04/fda_approves_ip ad_iphone_radiology_app_for_mobile_diagnoses

[12] Mim Software Inc., 2013. http://www.mimsoftware.com/products/mobile/

[13] J. R. Mitchell, et al., "A Smartphone Client-Server Teleradiology System for Primary Diagnosis of Acute Stroke," Journal of Medical Internet Research, Vol. 13, No. 2, 2011, p. e31.

[14] Mim Software Inc, 2013. https://mim-cloud.appspot.com/\#/viewing

[15] Osirix Imaging Software, 2013. http://www.osirix-viewer.com/

[16] Innovation Reports, "Smart Phones Allow Quick Diagnosis of Acute Appendicitis," 2009.

http://www.innovationsreport.com/html/reports/studies/s mart_phones_quick_diagnosis_acute_appendicitis_14456 2.html

[17] Auntminnie.com, "Rads Do Well Using Smartphones to Read CT, MR Images," 2011.

http://svc1.auntminnie.com/index.aspx?sec=sup\&sub=pac \&pag $=$ dis \&ItemID $=93897 \& w f=1$

[18] Diagnostic Imaging, "FDA Clears ResolutionMD for Android Devices," 2013.

http://www.diagnosticimaging.com/vendors/fda-clears-res olutionmd-android-devices

[19] Radiology Assistant, 2013. http://www.radiologyassistant.nl/en/p521123b084e65/and roid-app.html

[20] Tidal Pool Software, 2013. http://www.tidalpool.ca/radiationpassport/

[21] iMedical Apps Team, "SonoAccess Is a Free iPhone App with Great Tools for Learning Bedside Ultrasound," 2012. http://www.imedicalapps.com/2012/09/sonoaccess-iphon e-app-ultrasound/

[22] App Brain, "Rad-Rx," 2012.

http://www.appbrain.com/app/rad-rx/com.radrx.one.
[23] RSNA, 2013. http://myrsna.rsna.org/

[24] European Radiology, 2013. http://www.european-radiology.org/cms/website.php?id=/ en/index/news/data9910.htm

[25] RCR, 2013. http://www.irefer.org.uk/

[26] Universal Logbook, 2013. http://logbook.burgul.com/

[27] Mobi Health News, "FDA Approves Mobisante's Smartphone Ultrasound," 2011.

http://mobihealthnews.com/10165/fda-approves-mobisant es-smartphone-ultrasound/

[28] Mobisante, 2013. http://www.mobisante.com/

[29] Business Wire, "Matchbox Mobile Help Mobisante Win Award for Best M-Health Innovation at Mobile World Congress," 2013.

http://www.businesswire.com/news/home/201103240063 23/en/Matchbox-Mobile-Mobisante-Win-Award-M-Healt h-Innovation. Accessed October 2013

[30] Apple Inc., “Apple Answers the FCC's Questions,” 2012. http://www.apple.com/hotnews/apple-answers-fcc-questio ns/. Accessed August 2013

[31] US Food and Drug Administration, "Mobile Medical Applications," 2013.

http://www.fda.gov/downloads/MedicalDevices/.../UCM2 63366.pdf

[32] Kaiser Health News, "Lawmaker Pitches New FDA Office of Mobile Health," 2012.

http://www.kaiserhealthnews.org/Stories/2012/September /27/FDA-Mobile-apps.aspx?p=1

[33] D. Lightley, "Mobile Medical Applications: A Great Way of Reaching Healthcare Professionals?" Clinical Business Excellence, Vol. 1, No. 8, 2012, 3 p.

http://www.clinicalbusinessexcellence.co.uk/issue/8/CBE $\% 208$.pdf

[34] CISCO mConcierge, "BYOD Insights 2013: A CISCO Partner Network Study," 2013.

http://www.ciscomcon.com/sw/swchannel/registration/intenet $/$ registration.cfm?SWAPPID $=91 \&$ RegPageID $=3502$ 00\&SWTHEMEID=12949\&traffictype $=$ Direct

[35] K. Amin and A. Chandrasena, "The Uses of the iPhone for the Plastic Surgeon: Friend or Foe?" Plastic and Reconstructive Surgery, Vol. 129, No. 2, 2012, pp. 408e409e. http://dx.doi.org/10.1097/PRS.0b013e31823aeefa

[36] Teaching and Learning Research Programme, "Education 2.0? Designing the Web for Teaching and Learning," 2012. http://www.tlrp.org/pub/documents/TELcomm.pdf. 